\title{
Fano resonances in quasi-one-dimensional electron waveguides
}

\author{
Erkan Tekman* and Philip F. Bagwell \\ School of Electrical Engineering, Purdue University, West Lafayette, Indiana 47907
}

(Received 19 November 1992; revised manuscript received 11 February 1993)

\begin{abstract}
In the spectroscopy of atoms and molecules, an asymmetric Fano resonance arises whenever a bound state associated with one electronic configuration is coupled to the ionization continuum of a different configuration. A strikingly similar resonance appears for electronic transport in conductors with more than one subband, independent of the specific details of the system under study. We develop a two-subband approximation which describes the Fano resonances for conduction through an electron waveguide containing donor impurities, for $\Gamma-X-\Gamma$ intervalley tunneling in a GaAs $/ \mathrm{Al}_{x} \mathrm{Ga}_{1-x} \mathrm{As} / \mathrm{GaAs}$ heterojunction, and for an electron waveguide coupled to a resonant cavity. Interference between the direct and intersubband transmission channels gives rise to the asymmetric Fano resonance.
\end{abstract}

\section{INTRODUCTION}

Atoms and molecules have different electronic configurations. In the simplest treatment of an atom or molecule these different configurations do not interact, and their Schrödinger wave functions are approximately orthogonal. For certain transitions between high-lying energy levels, however, the electron-electron interaction between these different electronic configurations must be taken into account. The most interesting type of such a "configuration interaction" arises when one of the states belongs to an ionization continuum of energy levels, while the other is a discrete state. An electron in such a bound state can then decay into the continuum, moving away from the atom or molecule. Fano $^{1,2}$ showed that the asymmetric resonances observed in the inelastic-scattering spectra of light and electrons from atoms and molecules arise from these autoionization states. The same type of scattering resonances arise in nuclear physics. ${ }^{3}$

Recent theoretical studies of electron scattering from donor impurities in quasi-one-dimensional (quasi-1D) electron waveguides ${ }^{4-6}$ have revealed similar asymmetric resonance structures for conductance as a function of Fermi energy (or channel width). These asymmetric resonances appear when a continuum level from one subband is degenerate in energy with a bound state supported by a different subband, very similar to the atomic autoionization resonances. References 7-9 pointed out that the same asymmetric resonance feature also appears in other, seemingly unrelated physical systems. In addition to scattering from donor impurities in an electron waveguide,${ }^{4-6}$ the scattering from an oscillating barrier, ${ }^{7}$ optical absorption ${ }^{10}$ or electronic transport ${ }^{11}$ in "type-II" $\mathrm{GaAs} / \mathrm{Al}_{x} \mathrm{Ga}_{1-x} \mathrm{As} / \mathrm{GaAs}$ heterojunctions, transmission through a waveguide linked to a resonant cavity, ${ }^{12,13}$ transmission through a quantum-dot in magnetic field,,$^{8,14}$ and conduction through an array of disordered quantum wires ${ }^{15}$ all display the Fano resonances.
Each of these systems contains two different scattering channels, one belonging to a continuum and the other to a bound state.

In this paper we analyze the Fano resonances in quasi$1 \mathrm{D}$ systems. In Sec. II we develop a two-band model for the electronic transport in quasi-1D systems. Section III applies this two-band model to scattering from a donor impurity in an electron waveguide, ${ }^{4-6}$ tunneling through an $\mathrm{Al}_{x} \mathrm{Ga}_{1-x} \mathrm{As}$ barrier, ${ }^{11}$ and transmission through a waveguide linked to a resonant cavity. ${ }^{12,13}$ In Sec. IV we analyze how the Fano resonances are modified in more realistic, multiple-subband systems.

\section{THE TWO-BAND APPROXIMATION}

The Schrödinger equation describing scattering in a quasi-1D electron waveguide is ${ }^{16}$

$$
\left\{-\nabla^{2}+V_{c}(y)+V_{\mathrm{sc}}(x, y)\right\} \Psi(x, y)=E \Psi(x, y)
$$

where $V_{c}(y)$ is the confining potential in the lateral direction and $V_{\mathrm{sc}}(x, y)$ is the potential due to the scattering center. The geometry is shown in Fig. 1. We assume that the confining potential is uniform along the propagation direction, since any inhomogeneity can well be represented by an effective scattering potential. ${ }^{17}$ We are interested in scattering solutions $\Psi(x, y)$ for an electron wave incident from a single subband on the scatterer. As a result, there will be both transmitted and reflected waves, which may be either propagating or decaying as they move away from the scatterer.

For arbitrary $V_{\text {sc }}$ an analytical solution of Eq. (2.1) is not possible. Therefore, we make a simplifying assumption that the scattering potential is extremely localized along the direction of propagation, that is,

$$
V_{\mathrm{sc}}(x, y)=v(y) \delta(x)
$$




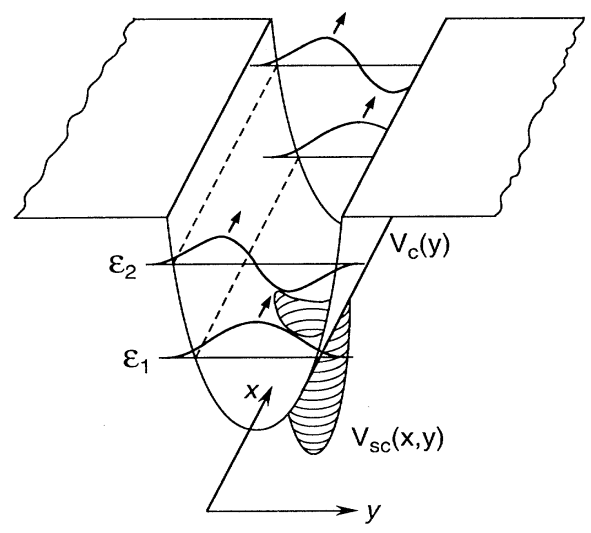

FIG. 1. Transmission through a donor impurity in an electron waveguide. A quasibound state produced near the locally attractive donor potential gives rise to Fano resonances in the waveguide conductance.

where $v(y)$ is an arbitrary function of the lateral coordinate. By expressing the scattering wave function $\Psi(x, y)$ in terms of the eigenstates $\left\{\phi_{m}\right\}$ of the confining potential $V_{c}$, Eq. (2.1) can be converted to a set of linear equations. The resulting matrix equation is ${ }^{5,6}$

$$
[-2 i \mathbf{K}+\mathbf{V}] \mathbf{T}=-2 i \mathbf{K},
$$

where $\mathbf{K}$ is the diagonal matrix of wave vectors $\mathrm{K}_{m n}=$ $k_{m} \delta_{m n}=\left(E-\epsilon_{m}\right)^{1 / 2} \delta_{m n}, \mathbf{V}$ is the interband transition matrix having elements $V_{m n}=\left\langle\phi_{m}|v(y)| \phi_{n}\right\rangle$, and $\mathbf{T}$ is the matrix of wave-function transmission amplitudes. The subband energies $\epsilon_{m}$ correspond to the eigenstates $\left\{\phi_{m}\right\}$.

The conductance can be calculated from the twoterminal Landauer formula, ${ }^{18}$

$$
G=\sum_{m n}\left(\frac{k_{m}}{k_{n}}\right) T_{m n}^{*} T_{m n}
$$

where the $m$ and $n$ are summed only over the propagating subbands. Here $T_{m n}$ denotes the amplitude of the transmitted wave in the $m$ th subband resulting from an incident wave in the $n$th subband. The unit of conductance in Eq. (2.4) is $2 e^{2} / h$.

The transmission amplitudes $\mathbf{T}$ can be extracted from Eq. (2.3) by inverting the matrix on the left-hand side. We do not discuss this general problem, since it usually requires numerical matrix inversion and has been studied by others. ${ }^{5-7}$ The simplest approximation to Eq. (2.3) is to neglect all but two subbands and solve the resulting $2 \times 2$ matrix equation. When $\epsilon_{1} \leq E \leq \epsilon_{2}$, so that the only incident wave is in the lowest subband, the reduced matrix equation for our scattering problem is

$$
\left[\begin{array}{cc}
-2 i k_{1}+V_{11} & V_{12} \\
V_{21} & 2 \kappa_{2}+V_{22}
\end{array}\right]\left[\begin{array}{l}
T_{11} \\
T_{21}
\end{array}\right]=\left[\begin{array}{c}
-2 i k_{1} \\
0
\end{array}\right],
$$

where the inverse decay length $\kappa_{2}$ is $k_{2}=i \kappa_{2}$. (We re- quire $\kappa_{2}>0$ to keep the wave function finite as $|x| \rightarrow \infty$.) The conductance is given by $G=\left|T_{11}\right|^{2}$ for $\epsilon_{1} \leq E \leq \epsilon_{2}$.

There exist three special points for the conductance $G$ (or equivalently $T_{11}$ ) in the complex energy plane; where $G(\mathrm{a})$ is zero, (b) is unity, and (c) has a pole. ${ }^{19}$ Inverting Eq. (2.5) shows that the transmission zero, $T_{11}=0$, is determined from $^{20}$

$$
2 \kappa_{2}+V_{22}=0 .
$$

Unity transmission amplitude $T_{11}=1$ occurs when

$$
2 \kappa_{2}+V_{22}=\frac{\left|V_{21}\right|^{2}}{V_{11}}
$$

The transmission pole $T_{11} \rightarrow \infty$ exists at the complex energy satisfying

$$
2 \kappa_{2}+V_{22}=\frac{\left|V_{21}\right|^{2}}{-2 i k_{1}+V_{11}} .
$$

The poles of $T_{11}$ or $G$ define the bound and quasibound states of the system as discussed in Ref. 7 .

\section{EXAMPLES OF FANO RESONANCES}

\section{A. Donor impurity in an electron waveguide}

This problem has been studied previously ${ }^{4-6,21,22}$ in the context of scattering in quasi-1D systems. By a donor impurity, we mean that $v(y)$ in Eq. (2.2) is negative as shown in Fig. 1 , so that $V_{11}<0$ and $V_{22}<0$. The predicted transmission $\left|T_{11}\right|^{2}$ from the two-band model is shown in Fig. 2. The asymmetric Fano resonance

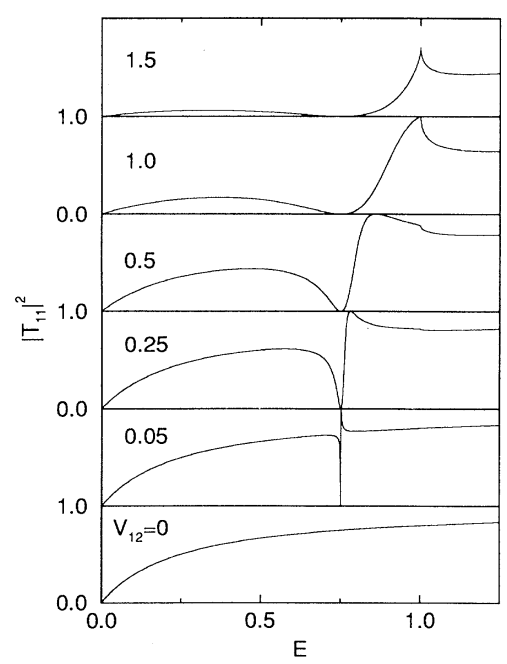

FIG. 2. Transmission probability as a function of energy for a donor impurity in an electron waveguide. A clear Fano resonance develops for weak intersubband interaction $\left(V_{12}=0.05\right)$, and gradually distorts as the intersubband scattering $\left(V_{12}=V_{21}\right)$ increases. The two subband energies are $\epsilon_{1}=0$ and $\epsilon_{2}=1$. The potential matrix elements are $V_{11}=V_{22}=-1$. 
is observed most clearly below a new subband opening when the intersubband matrix element $V_{12}$ is weak $\left(V_{12}=0.05\right)$. We now analyze the two-subband model to explain all the qualitative features of Fig. 2 .

In the absence of any intersubband interaction, the transmission amplitude $\tilde{T}_{11}$ from Eq. (2.5) is

$$
\frac{1}{\tilde{T}_{11}}=1-\frac{V_{11}}{2 i k_{1}}
$$

There is also a bound state in the second subband at an energy given by Eq. (2.6). We can write Eq. (2.6) as $\tilde{\kappa}_{2} \equiv$ $-V_{22} / 2$, which corresponds to an energy $E=\epsilon_{2}-E_{B}$. The binding energy is $E_{B}=\left(\tilde{\kappa}_{2}\right)^{2}$. Due to absence of interaction, this bound state is equivalent to that found for a strictly $1 \mathrm{D}$ problem. ${ }^{23}$ Therefore, in the absence of intersubband interaction, we have two types of states below $\epsilon_{2}$ : scattering states from the first subband and a bound state from the second subband.

The shape of the transmission resonance is determined by interference between the direct and intersubband transmission channels. Turning on the interaction between the subbands, the transmission amplitude becomes

$$
\frac{1}{T_{11}}=\frac{1}{\tilde{T}_{11}}+\frac{\left|V_{21}\right|^{2}}{4 i k_{1}} \frac{1}{\kappa_{2}-\tilde{\kappa}_{2}} .
$$

In addition to the direct transmission via the first subband (the first term), one has transmission mediated by the second subband (the second term). Noting that $1 / \tilde{T}_{11}$ has a negative imaginary part (when $V_{11}<0$ ), one easily finds destructive interference between the direct and intersubband transmission channels below the bound-state energy, i.e., for $\kappa_{2}>\tilde{\kappa}_{2}$. For energies above the bound state, the wave interference is constructive. At the bound-state energy the second term in Eq. (3.2) diverges, thus transmission vanishes.

As one further increases the energy beyond the bound state (decreases $\kappa_{2}$ ), unity transmission is obtained at a wave vector given by Eq. (2.7), that is, $\kappa_{2}=\tilde{\kappa}_{2}+$ $\left(\left|V_{21}\right|^{2} / 2 V_{11}\right)$. For very weak intersubband scattering, $V_{21} \simeq 0$, the transmission zero and unity transmission occur at almost the same energy. This gives the characteristic Fano line shape for the asymmetric transmission resonance. Stronger intersubband scattering increases the separation between the transmission zero and unity transmission points. When $V_{11} V_{22}=\left|V_{21}\right|^{2}$, the case for a $\delta$-function scatterer in the lateral direction, ${ }^{5}$ unity transmission takes place exactly at the onset of propagation for the second subband. A further increase in $\left|V_{21}\right|^{2} / V_{11}$, such that the second subband becomes propagating where unity transmission should occur, removes the unity transmission point altogether.

\section{B. $X$ valley tunneling in $\mathrm{Al}_{x} \mathrm{Ga}_{1-x} \mathrm{As}$}

A $1 \mathrm{D}$ band diagram for an $\mathrm{Al}_{x} \mathrm{Ga}_{1-x} \mathrm{As}$ barrier between two GaAs electrodes is shown in Fig. 3. By appropriately choosing the composition ratio of the barrier

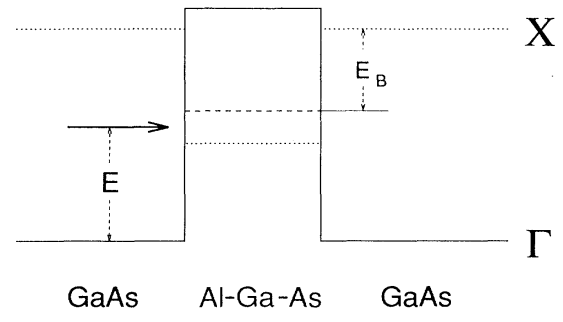

FIG. 3. Conduction-band edge for a GaAs- $\mathrm{Al}_{x} \mathrm{Ga}_{1-x} \mathrm{As}-$ GaAs single barrier tunneling structure. The alloy composition ratio in the barrier is chosen so that the $X$ valley minimum is lower than the $\Gamma$ valley minimum in the barrier, producing a quasibound state in the barrier.

alloy, the $X$ valleys can lie below the $\Gamma$ valley in the barrier. The transmission probability through such a barrier has previously been calculated. ${ }^{11}$ It was found that the transmission probability has asymmetric resonances, which were attributed to Fano-type effects.

Our two-band model may be used to approximate the tunneling through an $\mathrm{Al}_{x} \mathrm{Ga}_{1-x} \mathrm{As}$ barrier. Since the present treatment is only a crude approximation to the realistic problem, we intend no quantitative comparison with Ref. 11. For example, our model completely neglects the effect of the underlying atomic structure, considered through the use of the Bloch states in Ref. 11. In addition, the $\delta$ potential in Eq. (2.2) is only an approximation to the actual conduction-band structure. Nevertheless, we believe our two-band model captures the essential physics of $\Gamma-X-\Gamma$ intervalley tunneling.

The only essential difference between scattering from a donor impurity in a waveguide and $\Gamma-X-\Gamma$ intervalley tunneling is a sign change of the intrasubband matrix element $V_{11}$. Since the barrier potential is repulsive for the $\Gamma$ valley, we have $V_{11}>0$. The potential is still attractive for the $X$ valley, so that $V_{22}<0$ as before. Changing the sign of $V_{11}$ reverses the condition for destructive and constructive interference between the direct and intersubband transmission. This sign change therefore "inverts" the Fano resonance, as shown in Fig. 4. Equation (2.7) still describes the point of unity transmission, and Eq. (2.6) still determines the transmission zero. But the unity transmission point now occurs at a lower energy than the transmission zero. (The unity transmission obtained at $E=0$ for $V_{12}=1.0$ in Fig. 4 is an artifact of the two-band approximation, which disappears when other subbands are taken into account.)

One shortcoming of our two-band model is that more sophisticated calculations ${ }^{11}$ observe both types of Fano resonances shown in Figs. 2 and 4. This shortcoming is a consequence of our disregarding any crystal structure at the atomic scale. The form of the Bloch wave functions used in Ref. 11 (taken as a basis in which to expand the tunneling wave functions) may give rise to both positive and negative potential matrix elements $V_{11}$. The resulting resonance structure may vary therefore between Figs. 2 and 4. 


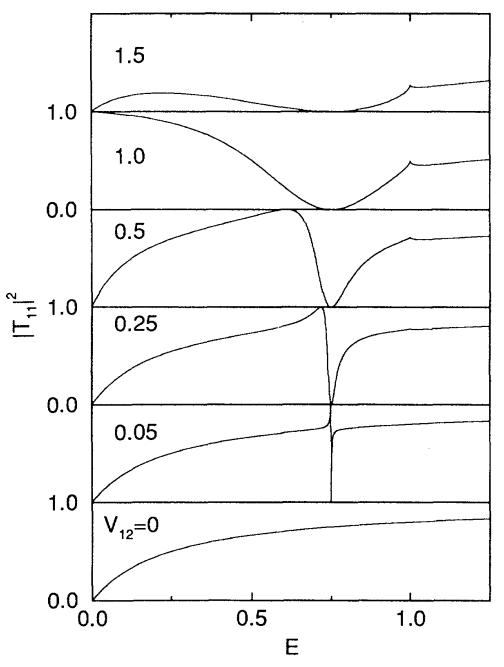

FIG. 4. Transmission probability as a function of energy for a two-band model of $\Gamma-X-\Gamma$ intervalley tunneling. Model parameters are the same as Fig. 2, except that $V_{11}=+1$. The Fano resonances are therefore "inverted" with respect to Fig. 2.

\section{Waveguide linked to a resonant cavity}

An electron waveguide is coupled to a resonator (a terminated stub of length $L$ ) via a tunneling barrier as shown in Fig. 5. References 12 and 13 obtained the conductance through this geometry by using the Landauer formula, ${ }^{18}$ finding transmission resonances as one changes either the energy of the incident wave or the length $L$ of the resonant cavity. Reference 13 also determined the pole-zero structure of the transmission coefficient in the complex energy plane, further clarifying the nature of the bound states.

Although the problem at hand is somewhat different than those studied above, it is possible to cast it into an equivalent two-band form. First, we discuss the solution

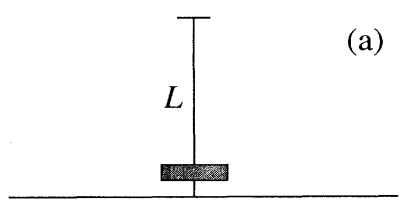

(b)

$\eta$

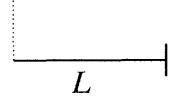

FIG. 5. (a) An electron waveguide coupled to a resonant cavity. $L$ is the length of the cavity and the gray box denotes a tunneling barrier determining the strength of waveguide-cavity coupling. (b) The equivalent two-band approximation. The waveguide produces the first band and the cavity the second band. The dotted line denotes the interaction strength $\eta$ between the subbands. of the Schrödinger equation for a network of quantum wires given in Ref. 24. Assuming that only the lowest subband is populated in all the wires (i.e., using strictly $1 D$ wires instead of Q1D ones), the wave function is equal to a scalar wave function $\psi_{e}$ along each edge $e$ of the network. These functions are determined by the matching conditions at the vertices $\{v\}$, and by the boundary conditions for incident and reflected waves far from the junctions. Since the electron probability distribution is a measurable entity, the wave function has to be single valued throughout the network. That is,

$$
\psi_{e_{1}}(v)=\psi_{e_{2}}(v)=\cdots=\psi_{e_{N}}(v),
$$

where $\left\{e_{1}, e_{2}, \ldots, e_{N}\right\}$ denote the edges joining at the vertex $v$. Similarly, conservation of charge is used to find a matching condition for the derivatives of the wave functions. Integrating the probability current density on a closed surface around $v$, one finds that

$$
\left.\nabla_{1} \psi_{e_{1}}\right|_{v}+\left.\nabla_{2} \psi_{e_{2}}\right|_{v}+\cdots+\left.\nabla_{N} \psi_{e_{N}}\right|_{v}=-i \lambda(v) \psi(v)
$$

where $\nabla_{i}$ denotes the derivative along the edge $e_{i}$, taken into the junction. In the original derivation ${ }^{24}$ of Eq. (3.4), $\lambda(v)$ is related to the scattering matrix for the junction. If one neglects the internal structure of the junction, on the other hand, the right-hand side of Eq. (3.4) denotes the presence of a $\delta$-function potential barrier of the form $V(v)=+i \lambda(v) \delta(v)$ at the junction.

Applying the matching technique described above to the system shown in Fig. 5(a), one gets the solution as found in Ref. 13. Instead, we modify the system to yield the two-band approximation. The approximate equivalent system is shown in Fig. 5(b). We take the electron waveguide as the first "subband," and consider the resonant cavity as the second "subband." The intersubband interaction between the waveguide and resonant cavity is introduced $^{25}$ using a dimensionless coupling parameter $\eta$, with $0<\eta<1$. Let $\psi_{\text {Stub }}$ denote the cavity wave function in the absence of any coupling to the waveguide, so that $\psi_{\text {Stub }} \sim \sin (k y)$ inside the cavity. To a good approximation, ${ }^{25}$ allowing the localized cavity state to leak into the waveguide increases the unperturbed wavefunction $\psi_{\text {Stub }}$ by $1 / \eta$ at the junction, and decreases its derivative by $\eta$. The matching conditions then become

$$
\psi_{L}(v)=\psi_{R}(v)=\frac{1}{\eta} \psi_{\mathrm{Stub}}(v)
$$

and

$$
\nabla \psi_{L}(v)+\nabla \psi_{R}(v)+\eta \nabla \psi_{\mathrm{Stub}}(v)=-i \lambda(v) \psi(v) .
$$

The coupling parameter $\eta$ thus scales the logarithmic derivative of the cavity wave function by a factor $\eta^{2}$ at the junction, ${ }^{26}$ namely, the logarithmic derivative of the wave function along the stub edge becomes

$$
\left.\frac{\nabla \psi}{\psi}\right|_{v}=\left.\eta^{2} \frac{\nabla \psi_{\text {Stub }}}{\psi_{\text {Stub }}}\right|_{v}=\eta^{2} k \cot (k L) .
$$


The two-band model above corresponds to the matrix equation

$$
\left[\begin{array}{cc}
-2 i k+i \lambda & -2 \eta i k \cos (k L) \\
-\eta i k & 2 k \sin (k L)
\end{array}\right]\left[\begin{array}{l}
T_{11} \\
T_{21}
\end{array}\right]=\left[\begin{array}{c}
-2 i k \\
0
\end{array}\right]
$$

The second row of Eq. (3.8) enforces continuity of the wave function (3.5), while the the first row enforces current conservation (3.6). In the limit $\eta \rightarrow 0$, the waveguide and cavity are completely decoupled. One then finds the transmission down a waveguide with an impurity, i.e., $\tilde{T}_{11}=(1-\lambda / 2 k)^{-1}$. The cavity, on the other hand, has a set of resonant states given by $k L=n \pi$. For nonzero $\eta$ the transmission amplitude becomes

$$
T_{11}=\frac{2}{(2-\lambda / k)+i \eta^{2} \cot k L} .
$$

When $\eta=1$ and $\lambda=0$, our result for transmission is equal to that found by Porod et al. ${ }^{13}$ for the "strongly coupled" case they studied. For this case, the interaction between the subbands (i.e., the branches) is purely geometrical and our approximate model becomes equivalent to that of Ref. 13. The transmission zeros and resonances are given by $k L=n \pi$ and $k L=(n+1 / 2) \pi$, respectively. For $\lambda=0$, Eq. (3.9) states that the interference between the direct and intersubband channels is always destructive. This is because the noninteracting waveguide always has unity transmission. The poles of Eq. (3.9) are given by (for $\lambda=0$ )

$$
k=\frac{n \pi}{L}-i \frac{1}{L} \tanh ^{-1}\left(\frac{\eta^{2}}{2}\right)
$$

which has the same real part as the transmission zeros. Increasing the intersubband interaction causes the poles to move away from the real axis, so that the quasibound states become more leaky.

The transmission probability $\left|T_{11}\right|^{2}$ as a function of energy is shown in Fig. 6. Increasing the interaction between the subbands causes the regions of near unity transmission to become narrower and the dips to become wider, in qualitative agreement with Refs. 12 and 13. Movement of the poles and zeros in the complex energy plane is consistent with this behavior. Since our present two-band model is only a crude approximation to the system of Refs. 12 and 13, we do not intend a quantitative comparison.

When we include additional scattering at the junction by having $\lambda \neq 0$, we find that the interference varies from constructive to destructive (as in Figs. 2 and 4). Including intrasubband scattering along the waveguide (in this approximation) also forces the real part of the poles away from $k L=n \pi$. Thus, in the presence of a barrier near the junction, our results even more closely resemble the ones of Ref. 13, further strengthening the justification for our two-band model.

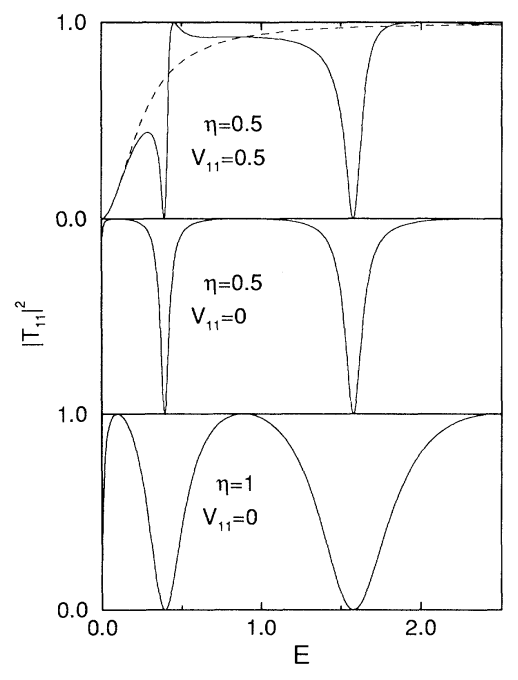

FIG. 6. Transmission probability versus energy for the electron waveguide coupled to a resonant cavity of length $L=5$. The lower two panels show different values of coupling strength $\eta$. Intrasubband scattering along the waveguide has also been taken into account in the upper panel, where $V_{11} \equiv+i \lambda=0.5$. A clear Fano line shape emerges with sufficient intrasubband scattering. The dashed curve in the upper panel corresponds to the case when the waveguide and resonant cavity are decoupled $(\eta=0)$.

\section{EFFECT OF THE OTHER SUBBANDS}

Real scattering problems cannot be quantitatively described using only two subbands. The effects of other configurations (which correspond to more than two subbands in quasi-1D systems) have been investigated by Fano ${ }^{1}$ and Mies, ${ }^{2}$ who called them "overlap" effects. Despite the differences with atomic systems, the Fanoresonance line-shape still survives in quasi-1D electronic systems in the presence of further intersubband interactions.

Figure 7 shows the conductance of the waveguide for the model of Sec. II when multiple subbands are present. Neither (a) additional propagating subbands, (b) additional evanescent subbands, or (c) both qualitatively alters the Fano line shape. Further, the Fano line shape is not very sensitive to the type of intersubband interaction. In Figs. 7(a) and 7(b) the interaction matrices are tridiagonal, so that only adjacent subbands interact, while Fig. $7(\mathrm{c})$ allows all the subbands to interact.

The presence of additional subbands can weaken the completely constructive and completely destructive interference present in the two-band model, removing the perfect unity or zero transmission. Including extra evanescent subbands also causes a lowering of the bound-state energy, shown in Figs. 7(b) and 7(c). However, the basic Fano line shape of the resonance remains qualitatively unchanged in the presence of multiple subbands.

Reducing the number of "subbands" to one eliminates the Fano resonance. If all transmitted waves must 


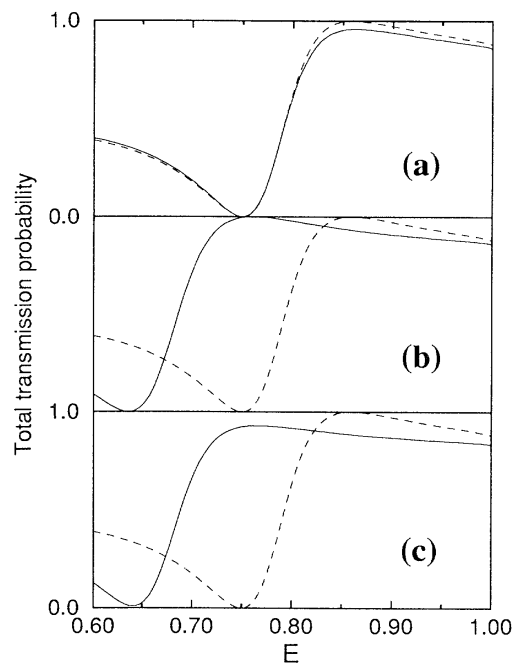

FIG. 7. Conductance versus energy through a multisubband waveguide. We consider (a) one decaying and five propagating subbands, (b) five decaying and one propagating subband, and (c) five decaying and five propagating subbands. The resonances are qualitatively unchanged by the presence of multiple bands. (The subband energies are $\epsilon_{n}=(n-1)$. The potential matrix elements are $V_{n n}=-1$ and $V_{n, n+1}=V_{n, n-1}=0.5$. For the lowest panel, the off-diagonal potential matrix elements are chosen as $V_{i j}=0.5 \exp (-2[|i-j|-1])$. The dashed curves show the result for the two-band approximation.)

pass through a resonant cavity, which is the case in a single-channel conductor, one obtains the standard resonant-tunneling transmission probability. The quasibound state in such a resonant cavity produces the pole (cross) shown in Fig. 8. For the Fano resonance, the electron can either transmit directly through the conductor or pass through some type of resonant state. The interfering amplitudes for the direct and bound-state transmission channels give rise to a transmission zero (circle) shown in Fig. 8. The combination of this pole-zero pair produces the asymmetric Fano line shape. Thus, the main feature which differentiates the Fano resonance from resonant tunneling is the existence of two independent transmission channels, only one of which passes through the quasibound state.

\section{CONCLUSION}

We developed a two-band model which adequately describes electronic transport through a donor impurity in an electron waveguide, $\Gamma-X-\Gamma$ intervalley tunneling in

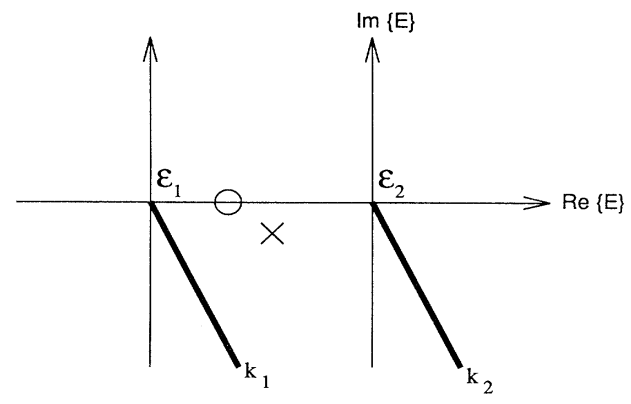

FIG. 8. Pole (cross) and zero (circle) structure of the transmission coefficient $T_{11}$ in the complex energy plane. The pole denotes a quasibound energy level, while the zero arises from an interference between direct and intersubband transmission channels. The combined pole-zero pair produces the characteristic Fano line shape. The thick lines denote the cut for the square-root function used to find the wave vector associated with each subband (Ref. 19).

a $\mathrm{GaAs} / \mathrm{Al}_{x} \mathrm{Ga}_{1-x} \mathrm{As} / \mathrm{GaAs}$ heterojunction, and transport in an electron waveguide coupled to a resonant cavity. Wave interference between the direct and intersubband transmission in quasi-1D electronic transport produce Fano resonances in the conductance versus Fermi energy or waveguide width. ${ }^{6}$ We further showed the Fano resonances arising in these systems to be robust to the inclusion of multiple subbands. Fano resonances can also occur in dirty electronic conductors, provided many such conductors are added in parallel. ${ }^{15}$ These facts should encourage experimental searches for Fano-type effects in multisubband electron-transport systems.

Besides the three physical systems emphasized in this paper, other systems display the Fano type of transmission resonance. In addition to transmission through an oscillating potential, ${ }^{7}$ the bound and resonant states associated with the Hall effect and bend resistance of quantum wire junctions ${ }^{8,14}$ also appear to be of the Fano type. Fano resonances seem to appear in low-dimensional electronic systems whenever a continuum level associated with one band is coupled to a discrete level from a second band.

\section{ACKNOWLEDGMENTS}

We thank Muhammad Alam for pointing out the Fano resonances and for discussions. We gratefully acknowledge financial support from the David and Lucile Packard Foundation. One of us (E.T.) is also grateful to the Scientific and Technical Research Council of Turkey.

\footnotetext{
* Permanent address: Department of Physics, Bilkent University, Bilkent 06533, Ankara, Turkey.

${ }^{1}$ U. Fano, Phys. Rev. 124, B1866 (1961). A simple discussion can be found in Sec. 2.10 of A.P. Thorne, Spectrophysics (Wiley, New York, 1974).

${ }^{2}$ F. H. Mies, Phys. Rev. 175, 164 (1968).

${ }^{3}$ J. M. Blatt and V. F. Weisskopf, Theoretical Nuclear
}

Physics (Wiley, New York, 1952). See Chap. IX, Fig. 2.2, and Chap. VIII, Figs. 8.2, 5.4, and 5.5.

${ }^{4}$ C. S. Chu and R. S. Sorbello, Phys. Rev. B 40, 5941 (1989).

${ }^{5}$ P. F. Bagwell, Phys. Rev. B 41, 10354 (1990); J. Phys. Condens. Matter 2, 6179 (1990).

${ }^{6}$ E. Tekman and S. Ciraci, Phys. Rev. B 42, 9098 (1990).

${ }^{7}$ P. F. Bagwell and R. K. Lake, Phys. Rev. B 46, 15329 
(1992). See also P. F. Bagwell, A. Kumar, and R. K. Lake, Scattering and Quantum Localization of Electrons by Static and Time-Varying Potentials, in Quantum Effect Physics, Electronics, and Applications, edited by K. Ismail, T. Ikoma, and H. I. Smith (IOP, London, 1992).

${ }^{8}$ J. U. Nöckel, Phys. Rev. B 46, 15348 (1992).

${ }^{9}$ P. J. Price (unpublished).

${ }^{10}$ K. Maschke, P. Thomas, and E. O. Göbel, Phys. Rev. Lett. 67, 2646 (1991).

${ }^{11}$ D. Y. K. Ko and J. C. Inkson, Semicond. Sci. Technol. 3, 791 (1988); J. P. Cuypers and W. van Haeringen, J. Phys. Condens. Matter 4, 2587 (1992); T. B. Boykin, B. Pezeshki, and J. S. Harris, Phys. Rev. B 46, 12769 (1992); D. Z. Y. Ting and T. C. McGill, ibid. 47, 7281 (1993).

${ }^{12}$ F. Sols, M. Macucci, U. Ravaioli, and K. Hess, J. Appl. Phys. 66, 3892 (1989).

${ }^{13}$ W. Porod, Z. Shao, and C. S. Lent, Appl. Phys. Lett. 61, 1350 (1992).

${ }^{14}$ H. U Baranger, Phys. Rev. B 42, 11479 (1990); H. Tamura and T. Ando, ibid. 44, 1792 (1991).

${ }^{15}$ A. Kumar and P. F. Bagwell, Phys. Rev. B 44, 1747 (1991).

${ }^{16}$ The Schrödinger equation (2.1) does not define unique length and energy units. The only relevant quantity is $\hbar^{2} / 2 m^{*}$, which is equal to $\sim 57 \AA^{2} \mathrm{eV}$ for GaAs. We take $\hbar^{2} / 2 m^{*}$ equal to unity in the analysis here. Thus, either the length or energy scale is fixed once the unit of the other is given. In our numerical examples one can take the unit of potential matrix elements to be $1 \mathrm{eV} \AA$, which yields an energy unit of $\sim 18 \mathrm{meV}$ and a length unit of $\sim 57 \AA$.

${ }^{17}$ E. Tekman and S. Ciraci, Phys. Rev. B 40, 8559 (1989); 43, 7145 (1991).

${ }^{18}$ M. Büttiker, Phys. Rev. Lett. 57, 1761 (1986); R. Landauer, J. Phys. Condens. Matter 1, 8099 (1989).

19 At this point, we must define the square root function we are using. Since the square root is not a single-valued function, it is necessary to have a cut in the complex energy plane. For each subband ( 1 and 2 in the two-band model) one has such a cut. The real (imaginary) part of $k_{n}$ has to be positive for $E>\epsilon_{n}\left(E<\epsilon_{n}\right)$ for real energy, in order to get physically acceptable scattering solutions. It is also clear that the imaginary part of the energy at the pole (the quasibound state) must be negative, so that the solutions decay in time. Putting all these considerations together, we conclude that the cut of the square-root function has to lie in the fourth quadrant of complex energy plane (i.e., for $\operatorname{Re}\{E\}>0$ and $\operatorname{Im}\{E\}<0)$, as shown in Fig. 8. Clearly, the exact position of the cut has to be chosen so that the pole lies in between the two cuts. The more conventional cuts along the negative or positive real axis are not acceptable since the former sweeps away the poles completely, and the latter (as well as the former) does not comply with the requirement that the conductivity is a continuous function of complex energy near the real axis.

${ }^{20}$ Equations (2.6)-(2.8) can also be expressed in terms of the electron energy. The transmission zero occurs at an energy $E=\epsilon_{2}-E_{B}$, where the binding energy $E_{B}$ is

$$
4 E_{B}=\left(\dot{V_{22}}\right)^{2} \text {. }
$$

Unity transmission is obtained at $E=\epsilon_{2}-E_{U}$, with

$$
4 E_{U}=\left(\frac{\left|V_{21}\right|^{2}}{V_{11}}-V_{22}\right)^{2}
$$

Equation (2.8) cannot be solved analytically to determine the complex energy pole. However, provided the bound state is not too near a subband edge, and that the binding energy is weak compared to the subband separation, this pole occurs at the energy $E \simeq \epsilon_{2}-E_{B}+i E_{I}$, with

$$
4 E_{I} \simeq \frac{V_{22}}{\left(\epsilon_{2}-\epsilon_{1}\right)}\left(2 V_{11} V_{22}-\left|V_{21}\right|^{2}\right) .
$$

${ }^{21}$ Y. B. Levinson, M. I. Lubin, and E. V. Sukhorukov, Phys. Rev. B 45, 11936 (1992).

${ }^{22}$ Ch. Kunze and R. Lenk, Solid State Commun. 84, 457 (1992).

${ }^{23}$ S. Gasiorowicz, Quantum Physics (Wiley, New York, 1974), p. 93.

${ }^{24}$ J. E. Avron, A. Raveh, and B. Zur, Rev. Mod. Phys. 60, 873 (1988).

${ }^{25}$ The introduction of the parameter $\eta$ is an approximation to the problem studied in Ref. 13. Consider the resonant cavity attached to a finite potential barrier, and in the absence of any interaction between the cavity and waveguide. A hard wall terminates the cavity at $y=0$, with a finite potential barrier at $y=L$. The cavity wave function for $0<y<L$ is then $\psi_{\text {Stub }}=A \sin (k y)$, with a logarithmic derivative $\nabla \psi_{\text {Stub }} / \psi_{\text {Stub }}=k \cot (k L)$ at $y=L$. This wave function will be matched to an exponentially decaying one in the barrier, giving rise to $\psi_{\text {Stub }}(y)=$ $A \sin (k L) e^{-\kappa(y-L)}$ and $\nabla \psi_{\text {Stub }}(y)=-\kappa A \sin (k L) e^{-\kappa y}$ for $L<y<\infty, \kappa$ being the inverse decay length in the barrier. The logarithmic derivative is therefore unchanged, so that $\nabla \psi_{\text {Stub }} / \psi_{\text {Stub }}=-\kappa=k \cot (k L)$ for $L<y<\infty$. When interaction between the cavity and the waveguide is turned on, i.e., the finite potential barrier composing one end of the cavity is lowered to form a portion of the waveguide, the wave function will have exponentially growing components in addition to exponentially decaying ones. The wave function for $L<y<(L+d)$, where $d$ is the thickness of the barrier, will become proportional to $\left(1+\alpha e^{2 \kappa y}\right) e^{-\kappa y}$. The derivative of the wave function is then proportional to $-\kappa\left(1-\alpha e^{2 \kappa y}\right) e^{-\kappa y}$. Here $\alpha$ is a small number, of the order of $e^{-2 \kappa d}$, which must be determined by the exact matching conditions for the problem. Instead of the complete solution (Ref. 13) for these new matching conditions, we develop approximate matching conditions (3.5) and (3.6). It is then possible to focus on the effects of the interaction, using a single parameter $\eta$, rather than the detailed mechanism of the interaction. From the previous paragraph, the result of interaction is to increase (decrease) the wave function (derivative) compared to the noninteracting case. For small values of $\alpha e^{2 \kappa d}$, which holds when the magnitude of the wave function inside the cavity is large (i.e., close to resonance), we may approximate the factor multiplying the wave function [see Eq. (3.5)] as $(1 / \eta)$ and the one multiplying the derivative [see Eq. (3.6)] as $\eta$. Hence $\left(1+\alpha e^{2 \kappa d}\right) \simeq 1 / \eta$ and $\left(1-\alpha e^{2 \kappa d}\right) \simeq \eta$. The logarithmic derivative for the interacting case becomes $\simeq \eta^{2} k \cot (k L)$, which is Eq. (3.7).

${ }^{26}$ The intersubband interaction used in the present approach is not completely realistic, since $\eta$ has to be a function of $k$ as noted above. In addition, we can use the present model only for $\eta \leq 1$ in conjunction with the approximation described above. Although the continuation for $\eta>1$ seems plausible from a mathematical point of view, it is not justifiable from a physical point of view. For example, for $\eta>\sqrt{2}$ one gets poles with $E_{I}>0$ from Eq. (3.10). 